\title{
Theory of Edge Plasma in a Spheromak
}

\author{
E.B. Hooper, R.H. Cohen, D.D. Ryutov \\ This paper was prepared for submittal to \\ 13th International Conference on Plasma Surface Interactions, \\ May 18-22, 1998, San Diego, CA
}

May 1998

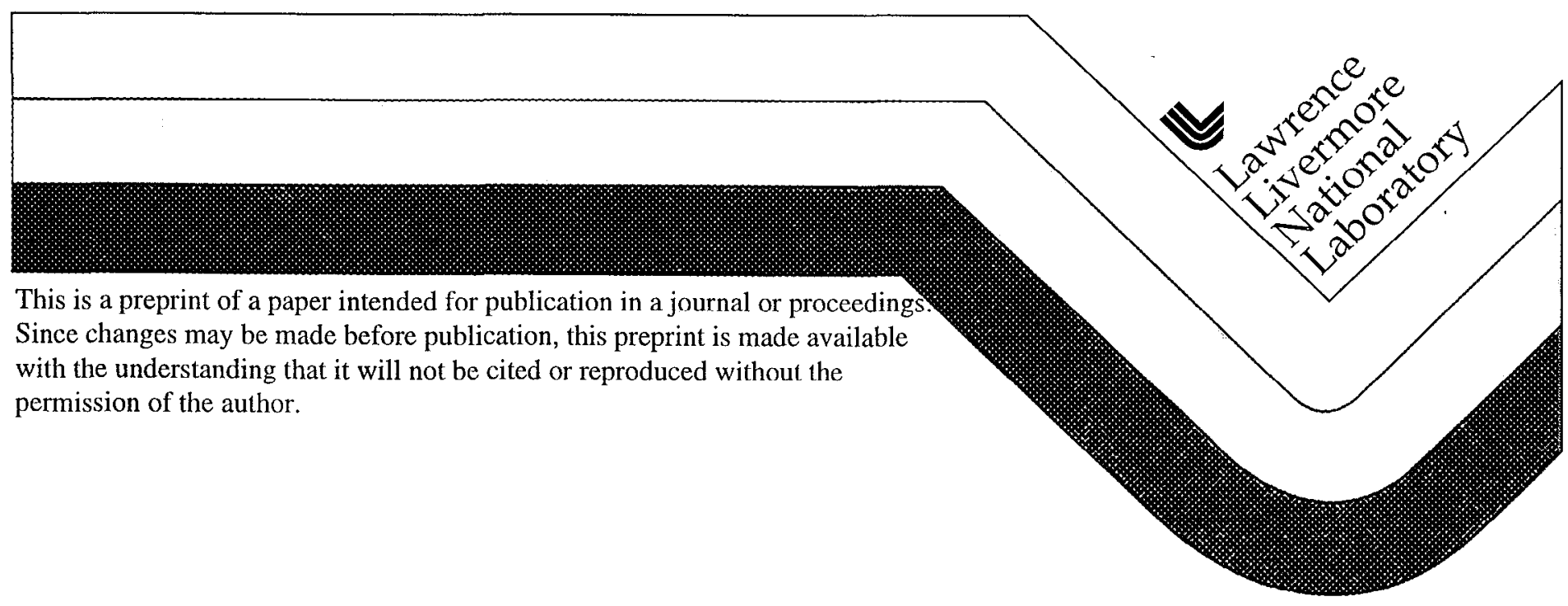




\section{DISCLAIMER}

This document was prepared as an account of work sponsored by an agency of the United States Government. Neither the United States Government nor the University of California nor any of their employees, makes any warranty, express or implied, or assumes any legal liability or responsibility for the accuracy, completeness, or usefulness of any information, apparatus, product, or process disclosed, or represents that its use would not infringe privately owned rights Reference herein to any specific commercial product, process, or service by trade name, trademark, manufacturer, or otherwise, does not necessarily constitute or imply its endorsement, recommendation, or favoring by the United States Government or the University of California The views and opinions of authors expressed herein do not necessarily state or reflect those of the United States Government or the University of California, and shall not bc uscd for advertising or product endorsement purposes 


\title{
Theory of edge plasma in a spheromak
}

\author{
E.B. Hooper, R.H. Cohen, D.D. Ryutov \\ Lawrence Livermore National Laboratory, Livermore, CA 94550
}

Properties of the edge plasma in the SSPX spheromak during the plasma formation and sustainment phases are discussed. For the breakdown and formation phase, the main emphasis is on the analysis of possible plasma contamination by impurities from the electrodes of the plasma gun (helicity injector). The issue of an azimuthally uniform breakdown initiation is also discussed. After the plasma settles down in the main vacuum chamber, one has to sustain the current between the electrodes, in order to continuously inject helicity. We discuss properties of the plasma on the field lines intersecting the electrodes. We conclude that the thermal balance of this plasma is maintained by Joule heating competing with parallel heat losses to the electrodes. The resulting plasma temperature is in the range of $15-30 \mathrm{eV}$. Under the expected operational conditions, the "current" velocity of the electrons is only slightly below their thermal velocity. Implications of this observation are briefly discussed.

\section{Introduction}

The attractiveness of spheromaks as fusion reactors stems from a topological simplicity of the reactor chamber and magnetic system, and relatively high plasma betas [1]. Based on this understanding, the SSPX (Sustained Spheromak Physics Experiment) project has been recently launched at LLNL. SSPX is a gun-driven spheromak, where the plasma is created (and then maintained) by a voltage applied between the electrodes of a coaxial helicity injector (gun) (Fig. 1). Our main purpose will be to study the processes in the edge plasma for the parameter domain characteristic for the SSPX device, although our results may be of interest for other spheromaks, too.

Plasma-wall interaction is important for essentially all types of fusion devices but in gun-driven spheromaks it may become a definitive element, especially at the early stages of the discharges. This is because both the formation of the toroidal configuration and its further sustainment requires direct contact of a plasma with the gun electrodes, and the current injected into the device depends on the properties of the edge plasma.

The scenario of SSPX operation is planned to be as follows: The quasi-steady solenoid (Fig. 1) creates a magnetic field of the shape shown in Fig. 1a. A typical value of the radial component of the magnetic field is $0.05 \mathrm{~T}$ (imposed magnetic flux $<34 \mathrm{mWb}$ ). The hydrogen (or deuterium) gas is then puffed into the gun region, in 8 points uniformly distributed over the azimuth and siluated on the tapered section of the gun. The voltage is applied between the inner and the outer electrodes with a controlled delay with respect to the gas-puff. Gas breakdown occurs, and a ring-shaped plasma is formed. Under the action of the azimuthal magnetic field, the plasma gets accelerated downward and is ejected into the main chamber. Rcconnection occurs in the zone near the gun muzzle, and the spheromak is formed (Fig. 1b). 
The second, quasi-steady-state phase of the experiment then begins, during which the spheromak is sustained by dynamo activity driven by the voltage applied between the electrodes, which amplifies the imposed flux by a factor $\sim 3-10$.

The external circuit that initiates the discharge consists of a condenser with a capacitance $C_{0}=10 \mathrm{mF}$ (initially charged to the voltage $\mathrm{V}_{0}=10 \mathrm{keV}$ ), an inductance with $L_{0}=2.12 \mu \mathrm{H}$, and a resistor $r_{0}=4 \mathrm{~m} \Omega$. After spheromak formation in the main chamber, the discharge is maintained for several milliseconds by a pulse-forming network (charging voltage $5 \mathrm{kV}$ ). The inner surface of the gun and the main chamber is coated with a $200 \mu \mathrm{m}$ thick tungsten coating.

\section{The initial stage of the discharge.}

The initial stage of the discharge, during which plasma stays inside the gun, is very important at least for two reasons: It determines the degree of the azimuthal uniformity of the initial configuration, and it may be definitive in establishing the impurity concentration inside the spheromak plasma (because during this phase the entire plasma is in immediate proximity to the gun electrodes). We divide this initial phase in two very easily distinguishable sub-phases: a relatively short one, when breakdown occurs and plasma reaches a considerable (say, 10\%) degree of ionization, while still remaining near the gas-puff position, and a longer one, during which the plasma gets moved towards the gun muzzle and ejected into the main confinement volume.

\subsection{The breakdown phase}

The mass of the hydrogen gas puffed into the gun will be approximately twice the mass of the plasma in the device at the nominal density $\sim 10^{14} \mathrm{~cm}^{-3}$. After the gas-puff occurs, one waits until the gas spreads out uniformly in the azimuthal direction: the gas from each valve would have to expand by the distance of approximately $2 \pi R / 8 \sim 35 \mathrm{~cm}$ (both ways). Assuming that the gas will spread at approximately the same speed in the vertical direction, the initial height $h_{0}$ of the gas cloud would be similar, $h_{0} \sim 35 \mathrm{~cm}$. In fact, because of the tapered shape of the outer electrode (Fig. 1), the gas penetration to the upper part of the gun will be limited so that $h_{0}$ will probably be closer to $h_{0} \sim 20 \mathrm{~cm}$. One can now evaluate the number density $n_{H_{2}}$ of the molecular hydrogen in the gas-puff: $2 \pi R h_{0} a\left(2 n_{H_{2}}\right)=2 n_{i} V$, where $a$ is the annulus width (below the valves), and $V$ is the volume of the plasma chamber. Taking $V \sim 0.4 \mathrm{~m}^{3}, h_{0} \sim 20 \mathrm{~cm}$, 
$a=16 \mathrm{~cm}, R=50 \mathrm{~cm}$, one finds that $n_{H_{2}} \sim 4 n_{i} \sim 4 \cdot 10^{14} \mathrm{~cm}^{-3}$. At this density, the product $a n_{H_{2}}$ corresponds to a breakdown voltage $\sim 7-8 \mathrm{kV}$ (the left branch of the Paschen curve, [2]).

The vertical distribution of the gas density has a maximum at some height (approximately corresponding to the position of gas valves). Because, as has been already pointed out, the gas density is at the left branch of the Paschen curve, the breakdown will first occur in the vicinity of this density maximum. The ionization rate is not a limiting factor: the efolding time for density growth is $\sim 1 / n_{H_{2}}<\sigma v_{i}>$; taking even a relatively low value for the ionization reactivity, $\sim 3 \cdot 10^{-9} \mathrm{~cm}^{4} / \mathrm{s}$, one finds that the exponentiation time is less than $1 \mu \mathrm{s}$.

The threshold energy above which the sputtering of the tungsten begins is $400 \mathrm{eV}$ for hydrogen, and $175 \mathrm{eV}$ for deuterium; at energy $\sim 1 \mathrm{keV}$ the sputtering coefficient is $\sim 4 \cdot 10^{-4}$ for hydrogen, and $\sim 7 \cdot 10^{-3}$ for deuterium. For $1 \mathrm{keV}$ oxygen it is approximately 0.2 [3]. The sputtering coefficient for $10 \mathrm{keV}$ ions is in the range of $\sim 2 \cdot 10^{-3}$ for the hydrogen, $\sim 10^{-2}$ for deuterium, and $\sim 0.4-0.5$ for oxygen [3]. Sputtering by hydrogen and deuterium is more important than the sputtering by heavier ions (e.g., oxygen) if the atomic concentration of the latter is less than $\sim 4 \cdot 10^{-3}$ in hydrogen and $\sim 2 \cdot 10^{-2}$ in deuterium. Throughout this paper, we will assume that this condition is satisfied and neglect the presence of gaseous impurities.

To eliminate impurities from the gas chamber to a maximum possible degrec, the whole chamber will be baked at a temperature up to $150 \mathrm{C}$ as well as glow discharge cleaned. In addition, following the initial stage of experiment, boronization of all the plasma-facing surfaces will be used. We believe that there is a good chance that the gaseous impurities (which may still remain at the remote surfaces in the diagnostic and vacuum port after all cleaning procedures [4]) will not reach the main chamber and the gun because of the pulsed nature of the experiment.

The other concern may be a heating of the tungsten surface in the gun which, under certain circumstances, may lead to formation of cathode spots and arcs [5]. Assume, for example, that the tungsten surface is heated for a time $\tau_{\text {HEAT }}$, and the energy delivered during this time per unit surface area is $Q$. Then, the surface temperature will rise by $\Delta T \sim Q / c_{\mathrm{V}}\left(\tau_{\text {HEAT }} \chi\right)^{1 / 2}$ where $\chi$ is the thermal diffusivity, and $c_{\mathrm{V}}$ is a thermal capacity per unit volume. The numerical form of this estimate is: $\Delta T(\mathrm{~K}) \sim 500 Q\left(\mathrm{~J} / \mathrm{cm}^{2}\right) /\left[\tau_{H E A T}(\mu \mathrm{s})\right]^{1 / 2}$. One can expect that, during the breakdown and translation phase, the wall heating will occur predominantly in a strip a few centimeters tall on the cathode surface in the area where the initial breakdown occurred: the magnetic field lines are frozcn into the cathode, and the ends of the current shell will probably remain attached to the zone where the current started to flow. The total energy dissipated in this ring at the cathode surface can be obtained by multiplying a characteristic current $(\sim 300 \mathrm{kA})$ by a characteristic sheath voltage $(\sim 100 \mathrm{~V})$ and the duration of the translation phase $(-100 \mu \mathrm{s})$. In this way, one finds that the energy released at the cathode 
surface is $\sim 3 \mathrm{~kJ}$. For the strip $5 \mathrm{~cm}$ tall this gives rise to a temperature increase $\sim 100 \mathrm{~K}$. This temperature increment is small and should not cause any problems. However, if the current exhibits filamentation instability and becomes concentrated over a smaller fraction of the surface, the temperature in the hot spots may increase considerably. Whether this undesirable phenomenon actually occurs, will become clearer after experiments begin.

Very soon after the initial breakdown occurs, the plasma density reaches the level $\sim 10^{8}$ $10^{9} \mathrm{~cm}^{-3}$ where the Debye radius evaluated for the applied voltage becomes smaller than the inter-electrode gap. At later times, the potential is concentrated in the Debye sheath near the cathode. The current density in the plasma at this stage is of the order of the ion saturation current density,

$$
j_{\text {sat }} \sim e n c_{\mathrm{s}}
$$

where $n$ is the plasma density, and $c_{s}$ is the ion acoustic velocity.

At the breakdown stage of the discharge, the following two simplifying factors help in a more quantitative analysis of the problem: first, the charge on the capacitor hasn't changed significantly yet and the voltage on it is very close to the initial voltage $V_{0}$; the current sheet hasn't yet moved much from the point where initiation occurred and, therefore, the inductance of the circuit remains constant. Thus, one can write the following equation for the voltage across the plasma gap:

$$
\mathrm{V}=\mathrm{V}_{0}-L_{0} \dot{I}_{\text {sat }}
$$

(the contribution of the external resistor $r_{o}=4 \mathrm{~m} \Omega$ is negligible during this stage).

The saturation current in the plasma can be evaluated as:

$$
I_{s a t} \sim 2 \pi R h j_{s u t}
$$

where $j_{\text {sat }}$ can be evaluated from Eq. (1), and $h$ is the height of the plasma shell. The timedependence of the density $n$ that enters Eq. (1) is determined by the rate of ionization processes. Note that, when all the neutrals are ionized, the saturation current is:

$$
I_{\text {sat }}^{(\max )}=2 e \mathrm{~N}_{\mathrm{H}_{2}} \mathrm{c}_{s} / a
$$

where $N_{\mathrm{H}_{2}}$ is the total number of hydrogen molecules injected into the device, and $a$ is the anode-cathode gap in the gun. Taking $N_{H_{2}} \sim 4 \cdot 10^{19}$, and $T=15 \mathrm{eV}$, one finds $I_{\text {sat }}^{(\max )} \approx 1.6 \mathrm{MA}$, well above the current ( $\sim 200-300 \mathrm{kA}$, see below) required to eject the plasma into the main volume. Therefore, the maximum saturation current is never reached during the brcakdown and translation phase.

We suggest using the following extrapolation to describe the current growth at an early stage of the discharge:

$$
I_{\text {sat }}=\varepsilon I_{\text {sat }}^{\max }[\exp (t / \tau)-1]
$$


The small parameter $\varepsilon$ (of which the final results depend only logarithmically) takes into account a low initial density, whereas $\tau$ characterizes the ionization time. We emphasize that approximation (5) is good only at the early stage of the discharge, when the ionization degree is still low. It does not describe the flattening of the $I(t)$ dependence which will inevitably occur later in the pulse but is sufficient to describe evolution of the current until the voltage drops, and the current reaches the level determined entirely by the external inductance (we will shortly find the time when this happens). From Eqs. (2) and (5) one finds the time-dependence of the voltage across the gun:

$$
V=V_{0}-\frac{\varepsilon L_{0} I_{s a t}^{\max }}{\tau} \exp \left(\frac{t}{\tau}\right)
$$

This voltage drops rapidly because of inductive effects. The time within which it reaches a level $\Delta \mathrm{V} \sim 200 \mathrm{~V}$ where the sputtering ceases to exist is:

$$
\iota_{1}=\tau \ln \frac{\mathrm{V}_{0} \tau}{\varepsilon L_{0} I_{\text {sat }} \max }
$$

(we have neglected $\Delta \mathrm{V}$ compared to $\mathrm{V}_{0}$ ). The plasma current at this point is $\mathrm{V}_{0} \tau / L_{0} \sim 15 \mathrm{kA}$ (for $\tau \sim 3 \mu \mathrm{s}$; the parameter $\tau$ depends on the ionization rate and may lie between 1 and $4 \mu \mathrm{s}$ ). Note that the plasma current doesn't depend on $\varepsilon$ at all. For the height $h$ of the plasma shell of the order of $4-5 \mathrm{~cm}$, this current corresponds to the plasma density $\sim 10^{13} \mathrm{~cm}^{-3}$. Taking $\varepsilon \sim 10^{-3}$, one finds: $t_{l} \approx 10 \mu \mathrm{s}$.

An upper-bound estimate for the amount of sputtered tungsten is:

$$
N_{Z}<\frac{S_{0}}{e} \int_{0}^{t_{1}} I_{s a t}(t) d t \approx \frac{S_{0} V_{0} \tau^{2}}{e L_{0}},
$$

where $S_{0}$ is the maximum sputtering coefficient, corresponding to the initial voltage. Taking $S_{0}=2 \cdot 10^{-3}$, and the other parameters as before, we find $N_{Z^{\sim 5}} 5 \cdot 10^{14}$. This should be compared with the total number of hydrogen ions in the system, $\sim 4 \cdot 10^{19}$. Hence, sputtering by plasma ions produces an insignificant amount of impurities in the breakdown phase.

The sputtered tungsten atoms have an energy in the range of a few electron-volts, and velocity $\sim 2 \cdot 10^{5} \mathrm{~cm} / \mathrm{s}$. Within a few microseconds, they will penetrate a distance of the order of $1 \mathrm{~cm}$ from the wall. Some of them will be ionized (the ionization time for tungsten atoms in a 10-20 eV plasma with a density $10^{13} \mathrm{~cm}^{-3}$ will be $\sim 10 \mu \mathrm{s}$ ) and accelerated back to the cathode. The self-sputtering coefficient for tungsten is $\sim 2$ at $10 \mathrm{keV}$, and that might cause a run-away of tungsten accumulation. However, the exponentiation time cannot be shorter than the ionization time $10 \mu \mathrm{s}$. This is the time when inductive effects become already important, and the sheath potential drops to $1-2 \mathrm{keV}$. At this lower voltage, the self-sputtering coefficient becomes less than 1 , and the runaway gets terminated. We therefore conclude that selfsputtering can hardly increase the amount of tungsten impurities by more than a factor of 2-3. 
As noted earlier, the breakdown voltage at the hydrogen density $n_{H 2} \sim 4 \cdot 10^{14} \mathrm{~cm}^{-3}$ (that will be established after azimuthal spreading of the gas-puffs) is rather high. If there are problems with discharge initiation at this density, one might consider applying voltage with a shorter time-delay with respect to the gas-puff. At a hydrogen density $\sim 10^{15} \mathrm{~cm}^{-3}$ the breakdown would require a considerably lower voltage, in the range of $1 \mathrm{keV}$. The problem in this case is that the current will be initiated in well-separated channels. If these channels persist in the later stages of the discharge, the plasma ejected into the main chamber may be too strongly non-uniform. The balance would be between a cleaner and a more uniform discharge. Alternatively, one could inject excess gas, which might lead to a lower quality spheromak.

\subsection{The plasma translation phase.}

Within a few microseconds following $t=t_{l}$ (Eq. (7)), almost complete plasma ionization occurs in the region where the breakdown has initially developed. As we show in Sec. 3, the plasma temperature $T$ will be in the range of $15-20 \mathrm{eV}$. According to Eq. (1), the current density will be $\sim 200 \mathrm{~A} / \mathrm{cm}^{2}$ (we assume that the ion density is $2 \cdot 10^{14} \mathrm{~cm}^{-3}$ ). This means that the current through a 1 -cm-tall ring at the surface of the gun electrode will be as high as $\sim 60$ $\mathrm{kA}$, and the nominal current of $300 \mathrm{kA}$ will flow through a ring $5 \mathrm{~cm}$ tall. In other words, a considerable skin-effect will occur. The electrical resistivity of a fully ionized 10-eV plasma is of the order of $10^{-3} \Omega \cdot \mathrm{cm}$. For the current density $\sim 200 \mathrm{~A} / \mathrm{cm}^{2}$, the electric field strength is only $0.2 \mathrm{~V} / \mathrm{cm}$. Therefore, the bulk resistance cannot change our conclusion regarding the presence of a strong skin effect.

Accordingly, one can expect that at this second stage the system will become almost purely inductive, and the potential drop over the sheath will become just a few T/e (needed to sustain the saturation current). Therefore, sputtering by hydrogen or deuterium ions will become negligibly small. Some sputtering by heavier impurities (oxygen, nitrogen) may still occur.

The current-carrying shell will be pushed downward and will collect the un-ionized hydrogen on its path. This will cause a further increase of the density of the current-carrying shell. Eventually, all the hydrogen will be swept by the shell.

The circuit equation at this later stage can be written as follows:

$$
\frac{d^{2}}{d t^{2}}\left[\left(L_{0}+L\right) I\right]+\frac{I}{C_{0}}=0
$$

where $L_{0}$ and $C_{0}$ are, respectively, the inductance $(2.12 \mu \mathrm{H})$ and capacitance $(10 \mathrm{mF})$ of the external circuit, whereas $L$ is the inductance of the plasma gun. The latter can be evaluated as: 


$$
L=L *\left(1+\varsigma \frac{z}{H_{g}}\right)
$$

where $z$ is the instantaneous position of the current shell measured from the position of the pulsed valves, $L^{*}$ is the inductance of the gun above the level of gas injection $\left(L^{*}=0.03 \mu \mathrm{H}\right)$, $\mathrm{H}_{\mathrm{g}}$ is a distance from the gun muzzle to an injection point, and $\zeta$ is a dimensionless parameter depending on the geometrical characteristics of the problem (in our case, $\zeta=2.5$ ).

The position of the current shell can be determined from the momentum balance equation:

$$
\frac{d}{d t}\left(\mu \frac{d z}{d t}\right)=2 \pi R a\left(\frac{B_{\varphi}^{2}}{2 \mu_{0}}-\eta \frac{B_{0}^{2}}{2 \mu_{0}} \frac{z}{H_{g}}\right)
$$

Here $\mu$ is the mass of the gas-puff incorporated into the motion (the current-carrying shell gradually collects the gas initially situated in front of it); the first term on the right-hand-side (r.h.s.) describes the accelerating pressure of the toroidal magnetic field, whereas the last term describes the restoring force acting from the side of the initial poloidal bias field that is being stretched in the vertical direction by the shell progressing to the gun muzzle. The numerical factor $\eta$ describes the degree of stretching; in our case it is equal to 4-5 (see the geometry of the gun in Fig. 1). We used a linear approximation to the $z$-dependence of the restoring force. This is certainly correct at not-too-large $z$ 's; the full dependence can be found only as a result of extensive numerical analyses of the equilibria.

Under conditions of our experiment, the inertial force on the left-hand-side of Eq. (12) is small compared to the driving force described by the first term on the r.h.s. and can be neglected. Accordingly, the motion of the plasma shell in the gun will be a sequence of equilibrium states determined by the instantaneous value of the toroidal magnetic field, $B_{\varphi}=\mu_{0} I / 2 \pi R$. Taking $B_{0}=0.05 \mathrm{~T}$, and $\eta=5$, one finds that the plasma shell reaches the gun muzzle $\left(z=H_{g}\right)$ at the current $I \approx 300 \mathrm{kA}$.

As the gun inductance is small, one can neglect it in Eq. (9). The current then follows a simple sinusoidal dependence,

$$
I=\frac{\mathrm{V}_{0} \sqrt{L_{0} C_{0}}}{L_{0}} \sin \left(\frac{t}{\sqrt{L_{0} C_{0}}}\right)
$$

The maximum would be reached at $t=200 \mu \mathrm{s}$ and would be equal to $700 \mathrm{kA}$ although the circuit and plasma resistance will limit it (note that Eq. (12) can be used for the first 100 or so microseconds; after that, the current is sustained at an approximatcly constant level by a pulseforming network). The current $300 \mathrm{kA}$ will be reached at approximately $70 \mu \mathrm{s}$. During these 70 
$\mu \mathrm{s}$, there will be some voltage drop over the gun (caused by the changing current and the gun inductance). At the insulator, it will be equal to $d / d t\left(L^{*} I\right)$, and will drop to zero at the plasma surface. From the above equations, one easily finds that, at the time when the plasma reaches the gun muzzle, $d / d t(L * I) \sim 1.5 \mathrm{kV}$. This voltage is applied predominantly in the upper part of the gun, where the plasma density is low; therefore, one shouldn't expect a considerable influx of tungsten during this phase.

After the plasma shell reaches the end of the gun, it is ejected into the main vacuum volume. This plasma bursting occurs at the inertial time-scale $\tau_{\text {burst }}[6]$; in our case, $\tau_{\text {lurst }}$ will be a few microseconds. A rapid change of the inductance (at this stage, the approximation (10) is

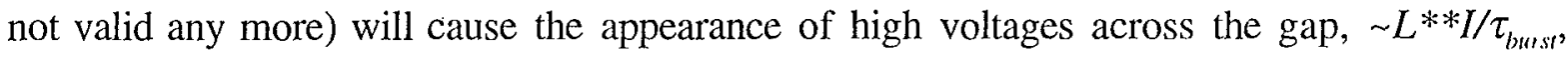
where $L^{* *}$ is the inductance of the main plasma volume, $\sim 0.1 \mu \mathrm{H}$. Taking $I=300 \mathrm{kA}$, and $\tau_{\text {burst }}$ $\sim 5 \mu$, one finds that the gun voltage may reach $\sim 5 \mathrm{kV}$. This may cause additional sputtering, which, however, will occur well behind the plasma blob that has been already translated into the main volume. The estimate from above for the number of tungsten atoms released in the gun is: $N_{Z}<S \cdot I \cdot \tau_{\text {burs }} /$ e. For $S \sim 10^{-3}$, one finds that $N_{Z}<10^{16}$. Although this number is relatively large, one should remember that, first, the estimate is made with a very large margin and, second, that these atoms will be released in the gun, and most of them will be redeposited there, without entering the main volume.

The plasma current and the voltage at the gun insulator is shown in Fig. 2 for a resistivc plasma model and the actual power system.

\section{Plasma on the open field lines during the self-sustained phase.}

After some transitional period, the plasma blob will settle down in the main chamber, and a quasi-steady-state phase will begin. The expected duration of this phase is several milliseconds. During this stage, the current originating in the gun will drive a reconnection process that will sustain and enhance the poloidal flux. The magnetic field configuration acquires the shape shown in Fig. 1b. We discuss now the plasma parameters on the field lines beginning and ending in the gun.

The current on these field lines will be in the range of $200 \mathrm{kA}$. From geometric considerations (see Fig. 1b), one can see that the parallel current density on these field lines will be as high as $1.5 \mathrm{kA} / \mathrm{cm}^{2}$. The relative velocity $u$ of electrons and ions is determined by the current density $j$ and plasma density $n$ :

$$
u(\mathrm{~cm} / \mathrm{s})=6 \cdot 10^{18} \mathrm{j}\left(\mathrm{A} / \mathrm{cm}^{2}\right) / n\left(\mathrm{~cm}^{-3}\right) .
$$

This yields $u$ of order of $10^{8} \mathrm{~cm} / \mathrm{s}$, much greater than the ion thermal velocity, $v_{T i}=5 \cdot 10^{6} \mathrm{~cm} / \mathrm{s}$ (deuterium, $20 \mathrm{eV}$ ), and comparable with the electron thermal velocity, $\mathrm{v}_{T e}=2 \cdot 10^{8} \mathrm{~cm} / \mathrm{s}$. 
If generation of electrons on the electrode walls (via the secondary and photo-emission) is small, then the parallel (to the magnetic field) current density cannot exceed the ion saturation current, which is of order of $e n \mathrm{v}_{T i}$, and the plasma would not be able to sustain the required current on the open field lines. On the other hand, in previous spheromak experiments, no obvious limitations on the current have been noticed. Therefore, one has to assume that there is either a strong electron emission from the walls, or the plasma density near the walls is considerably higher than in the rest of the open field line region, or both. Our present understanding is that the dense cold plasma near the walls will be formed almost inevitably (see below). The presence of the dense plasma near the electrodes may provide conditions where the current in the central (low density) part of the flux tube is carried by the electrons, while near the electrodes the current does not exceed the ion saturation current (because of a high plasma density there).

Emission of electrons from the walls can occur via the photo-effect (under the action of the $10-20 \mathrm{eV}$ photons emitted by the plasma) and via thermo-ionic and field emission from the micro-spikes and other microstructures. The intensity of the radiation losses sufficient for maintaining a current of $\sim 200 \mathrm{kA}$ is $\sim 40 \mathrm{MW}$, a few times less than the total power circulating in the plasma (we assume that the photoemission coefficient is $\sim 0.1$ ). In the plasma gun, the field lines intersect its surface at a small angle. This also helps to keep the required normal component of the current density on the electrode at a reasonable level $\sim 100 \mathrm{~A} / \mathrm{cm}^{2}$.

For the connection length around the spheromak $L \sim 2-3 \mathrm{~m}$, the acoustic time $L / c_{s}$ is of the order of $10^{-4} \mathrm{~s}$, considerably shorter than the pulse length. Accordingly, the plasma should be in a static equilibrium along the field lines. In the strongly collisional regimes typical for the edge plasma, one should expect only slow plasma flows along the field lines, with a considerable recycling of the neutrals. Therefore, we adopt as the model the pressure equilibrium along the field lines,

$$
p=2 n T=\text { const }
$$

Note that possible "wobbling" of the flux-tube in the course of the dynamo activity does not affect this conclusion if the motions occur with a speed below the sound speed.

The Joule heating provides a mechanism, which, in principle, can maintain plasma both in the central column and in the area near the outer wall. Let us define a characteristic Joule heating time as the time within which the thermal energy content is doubled by the Joule heating,

$$
\tau_{j}=(3 n T) /\left(j^{2} / \sigma\right)
$$

Numerically,

$$
\tau_{f}(s)=6 \cdot 10^{-18} n(\mathrm{~cm})^{-3}[T(\mathrm{eV})]^{5 / 2} /\left[j\left(\mathrm{~A} / \mathrm{cm}^{2}\right)\right]^{2} Z_{e f f}
$$


For $T=20 \mathrm{eV}, n=10^{14} \mathrm{~cm}^{-3}, Z_{e f f}=2$, and $j \sim 1.5 \mathrm{kA} / \mathrm{cm}^{2}$, we find that $\tau_{j} \sim 3 \cdot 10^{-7} \mathrm{~s}$, by far shorter than the Bohm diffusion time even over the scale of a few mm. So, probably, the Joule heating is the main mechanism for sustaining the thermal equilibrium of the plasma on the open field lines.

Assuming that the main channel of the heat losses is parallel thermal conductivity, one can write the following balance equation:

$$
\frac{\partial}{\partial s} \kappa_{\|} \frac{\partial T}{\partial s}+\frac{j^{2}}{\sigma}=0
$$

where $\kappa_{\| /}$is electron thermal conductivity and $s$ is a distance along the field line. In Eq. (17), we assume that the magnetic field (and, therefore, the cross-section of the flux tube) do not vary along the field line. Assuming, for simplicity, symmetry of the problem with respect to the mid-point of the flux tube, one finds the following relation for the plasma temperature in the equatorial plane:

$$
\frac{j^{2} L^{2}}{\sigma_{0} \kappa_{\| 0} T_{0}}=\frac{16}{49}\left[\int_{0}^{1} \frac{d z}{\sqrt{1-z^{4 / 7}}}\right]^{2}=2.2
$$

where the subscript " 0 " designates the values of the corresponding quantities in the equatorial plane. Eq. (18) yields the numerical estimate for the plasma temperature:

$$
T_{o}(e V)=0.25\left[j^{2}\left(A / \mathrm{cm}^{2}\right) L^{2}(\mathrm{~cm}) Z^{2}{ }_{e f f}\right]^{1 / 5}
$$

For $j=1.5 \mathrm{kA} / \mathrm{cm}^{2}, L=200 \mathrm{~cm}, Z_{\text {eff }}=2$, we find $T_{0}=30 \mathrm{eV}$. At $T_{c}=30 \mathrm{eV}$, the thermal conduction time and the Joule heating time are of the same order. Note a relatively weak dependence of the temperature on all other parameters: this is because of the rapid decrease (increase) of the plasma thermal conductivity (electrical conductivity) with temperature. The estimate (19) predicts the value of $\mathrm{T} \sim 15 \mathrm{eV}$ for the gun phase of a spheromak (after the full ionization occurred).

Near the wall, the temperature (as determined from Eq. (17)) varies according to equation

$$
T \sim(\Delta s)^{2 / 7}
$$

where $\Delta s$ is a distance from the wall along the field line. The pressure equilibrium condition (14) then means that the density formally diverges at the wall, $n \sim(\Delta s)^{-2 n}$. This does not cause the divergence of the total number of plasma particles (as the density singularity is integrable) but it may cause some increase of radiative losses. 


\section{Summary}

We considered the physics of edge plasmas in gun-driven spheromak. The early stage of the discharge, when the plasma is situated inside the gun, determines the degree of symmetry of the initial spheromak plasma, and the amount of impurities scooped by the plasma from the walls of the gun. We argue that, in order to have good initial symmetry, it is advisable to let the neutral gas injecled by pulsed valves spread over the azimuth. We find that the breakdown voltage for the uniformly distributed plasma is relatively high, $\sim 5 \mathrm{kV}$. Still, it is by a factor of 2 below the initial applied voltage.

The amount of tungsten produced by sputtering during the breakdown stage is small (of the order of $10^{-3} \%$ of the plasma ions). The breakdown phase is followed by the translation phase, during which the plasma shell moves towards the end of the gun. At this stage, the voltage drop over the plasma is small, and no significant sputtering occurs in the region of a considerable plasma density. Some sputtering may occur way back near the gun insulator but it will hardly affect the main plasma. At the moment when the plasma bursts from the gun to the main experimental volume, a high inductive voltage $\sim 5 \mu \mathrm{s}$ in duration develops across the gun. It may cause sputtering by the ions of the residual plasma left in the gun. The upper estimate of the tungsten atoms produced at this instant is in the range of $0.1 \%$ of the plasma ions, but these atoms are produced inside the gun and will be redeposited on the walls of the gun.

In the quasi-steady state, the sustainment current flows in the edge plasma region, where the magnetic field lines begin and end in the gun (or on the walls of the main chamber). The thermal balance of this plasma is maintained by Ohmic heating vs parallel heat losses to the walls. The resulting plasma temperature $(\sim 15-20 \mathrm{eV})$ very weakly depends on the parameters of the problem.

\section{Acknowledgments}

This work was carried out under the auspices of the U.S. Department of Energy by Lawrence Livermore National Laboratory under Contract W-7405-ENG-48. 


\section{References}

1. E.B. Hooper, J.H. Hammer, C.W. Barnes, J.C. Fernández, F.J. Wysocki. Fusion Technology, 29, 191 (1996).

2. Yu.P. Raizer. “Gas Discharge Physics," Springer-Verlag, 1991.

3. C.F. Barnett, et al. (eds), Atomic Data for Fusion, Oak Ridge National Laboratory Report ORNL-6086, July 1990.

4. D. Guilhem et al. Paper O-10 presented at the 13th Intern. Conf. on Plasma-Surface Interaction in Fusion Devices, San Diego, May 1998.

5. "Electrical Breakdown in Gases," J.M. Meek and J.D. Craggs, Eds. John Wiley \& Sons, 1978.

6. C.W. Barnes, T.R. Jarboe, G.J. Marklin, S.O. Knox, I. Hennis. Phys. Fluids, B2, 1871 (1990). 


\section{Figures}
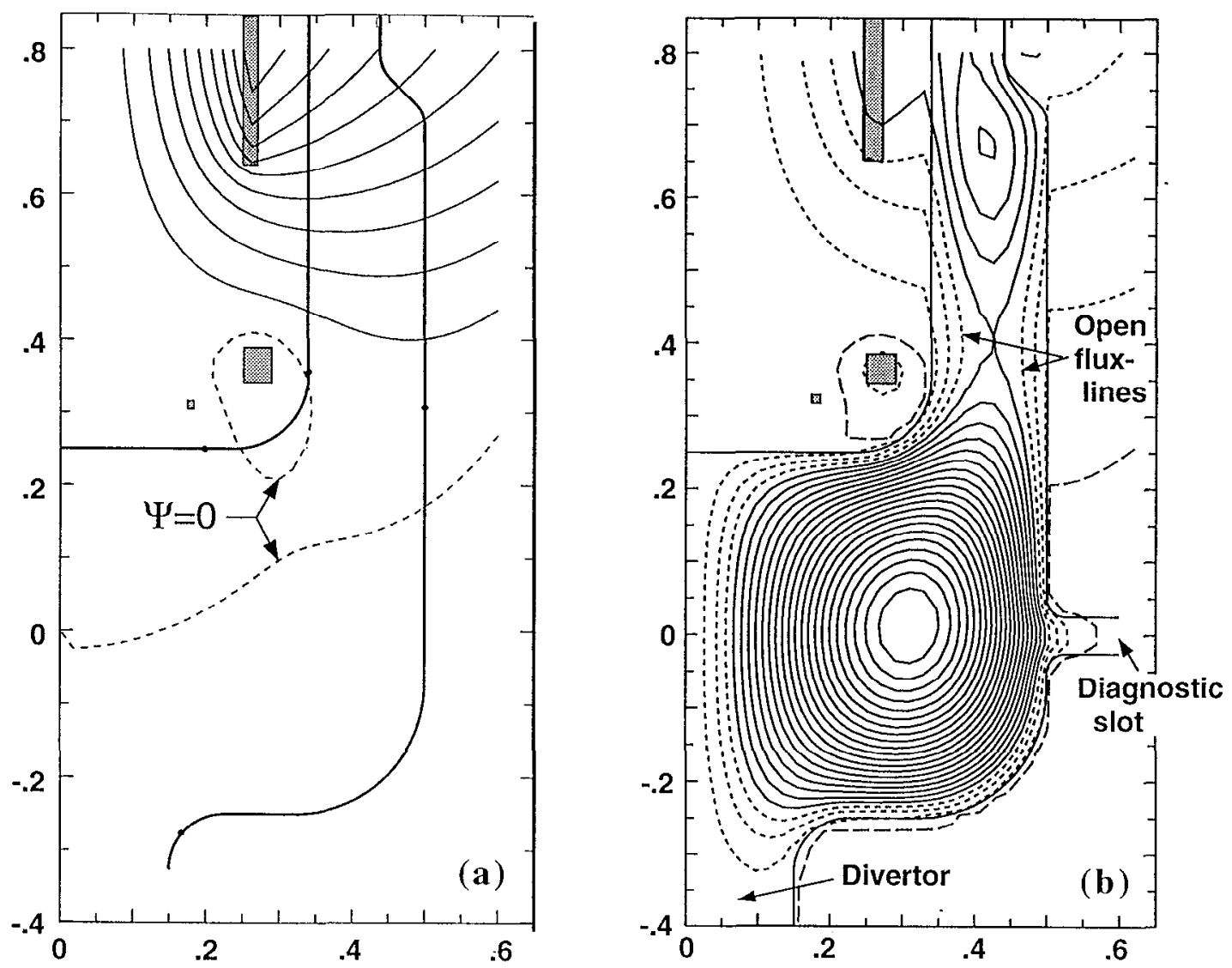

Fig. 1. The spheromak geometry and magnetic field configuration, axisymmetric around the left axis. Fig. 1a shows the vacuum magnetic field, generated by the magnetics shown in gray. Fig. $1 \mathrm{~b}$ is an example of the ideal MHD equilibrium of the coupled spheromak and gun. 

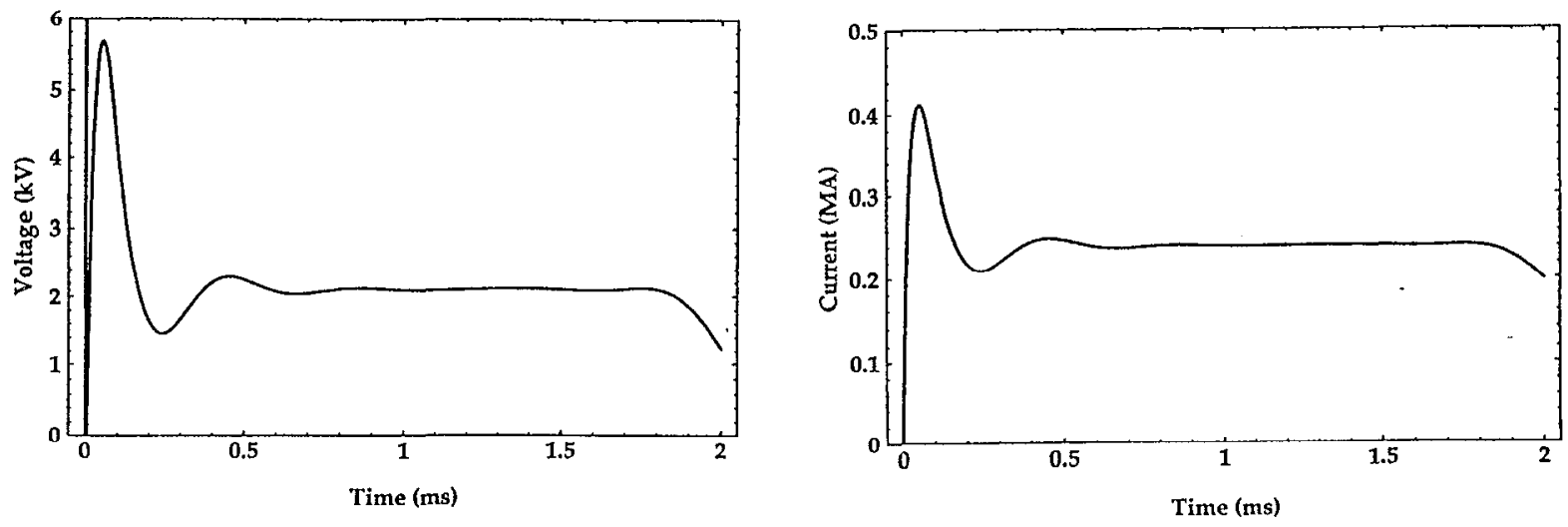

Fig. 2. An example of a model of the SSPX voltage and current, including the effects of coupling to the confined plasma. 


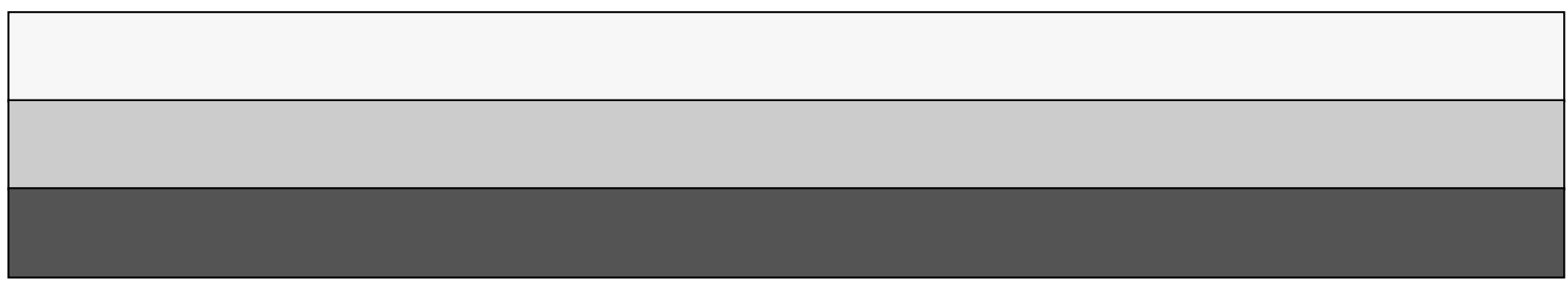

